INPLASY

PROTOCOL

To cite: Clemente et al.

Relationships between sleep, athletic and match

performance, training load and injuries: A systematic review in soccer players. Inplasy

protocol 202150029. doi:

10.37766/inplasy2021.5.0029

Received: 07 May 2021

Published: 07 May 2021

Corresponding author:

Filipe Manuel Clemente

filipe.clemente5@gmail.com

Author Affiliation:

Escola Superior Desporto e Lazer, Instituto Politécnico de Viana do Castelo, Rua Escola Industrial e Comer-cial de Nun'Álvares, 4900-347 Viana do Castelo, Portugal.

Support: None.

Review Stage at time of this submission: Data analysis.

Conflicts of interest:

None declared.

\section{Relationships between sleep, athletic and match performance, training load and injuries: A systematic review in soccer players}

\author{
Clemente, FM1; Afonso, J2; Costa, J3; Oliveira, R4; \\ Pino-Ortega, J4; Rico-González, M55.
}

Review question / Objective: The purpose of this systematic review was to summarize the evidence regarding the relationships between sleep and (i) athletic and match performance; (ii) training load; and (iii) injuries in soccer players.

Condition being studied: Sleep quality and quantity in soccer players.

Main outcome(s): Relationships between objective and/or subjective sleep measures (e.g., number of hours of sleep; quality of sleep) and one of the following measures: (i) athletic performance (e.g., cardiorrespiratory fitness, anaerobic power, strength/power, mobility, speed, agility); (ii) match performance (e.g., cognitive performance, tactical/technical performance; match-running performance); (iii) training load (measures related to psychophysiological or physical load); and/or (iv) injury occurrence.

INPLASY registration number: This protocol was registered with the International Platform of Registered Systematic Review and Meta-Analysis Protocols (INPLASY) on 07 May 2021 and was last updated on 07 May 2021 (registration number INPLASY202150029).

\section{INTRODUCTION}

Review question / Objective: The purpose of this systematic review was to summarize the evidence regarding the relationships between sleep and (i) athletic and match performance; (ii) training load; and (iii) injuries in soccer players. 
Condition being studied: Sleep quality and quantity in soccer players

\section{METHODS}

Search strategy: Electronic databases (EBSCOhost (SPORTDiscus), PubMed, Scielo, Cochrane, FECYT (Web of Sciences, CCC, DIIDW, KJD, MEDLINE, RSCI, and SCIELO)) were searched for relevant publications on March 29, 2021. Keywords and synonyms were entered in various combinations in title and/or abstract, with two code lines for free terms (soccer OR football) AND ("sleep*") AND one code line for MeSH terms (performance OR injur* OR load) NOT ("American football" OR "Australian football" OR rugby OR "Gaelic football"). Additionally, the reference lists of the studies retrieved were manually searched to identify potentially eligible studies not captured by the electronic searches.

Participant or population: Soccer players from any age group, competitive level or sex.

Intervention: Players monitored for sleep quality and/or quantity.

Comparator: Not mandatory. If available: comparisons between players with regular sleep ( $>7$ hours) and players identified with sleep depreviation and/or sleep loss (insomnia).

Study designs to be included: No restrictions with regard to study design.

Eligibility criteria: Inclusion criteria: (i) Soccer players from any age group, competitive level or sex; (ii) Players monitored for sleep quality and/or quantity; (iii) Not mandatory. If available: comparisons between players with regular sleep ( $>7$ hours) and players identified with sleep depreviation and/or sleep loss (insomnia); (iv) Relationships between objective and/or subjective sleep measures (e.g., number of hours of sleep; quality of sleep) and one of the following measures: (a) athletic performance (e.g., cardiorrespiratory fitness, anaerobic power, strength/power, mobility, speed, agility); (b) match performance (e.g., cognitive performance, tactical/technical performance; match-running performance); (c) training load (measures related to psychophysiological or physical load); and/ or (d) injury occurrence; (v) Only original studies (not restricted to any language). Exclusion criteria: (i) Sports other than soccer (e.g., futsal, beach football, basketball, rugby, Australian football), physical education students, general population; (ii) Non controlled for sleep quality and/or quantity; (iii) Sleep disorders other than sleep deprivation and/or sleep loss (e.g., sleep apnea, restless legs syndrom, narcolepsy); (iv) Other measures not related to athletic and match performance, training load and injury occurrence (e.g., wellness measures). No effect of the exercise in sleep quality or quantity. Studies that analysed the effect of exercise in sleep quality or quantity; (v) Other article types (e.g., reviews, letters to editors, trial registrations, proposals for protocols, editorials, book chapters and conference abstracts).

Information sources: Only original studies (not restricted to any language).

Main outcome(s): Relationships between objective and/or subjective sleep measures (e.g., number of hours of sleep; quality of sleep) and one of the following measures: (i) athletic performance (e.g., cardiorrespiratory fitness, anaerobic power, strength/power, mobility, speed, agility); (ii) match performance (e.g., cognitive performance, tactical/technical performance; match-running performance); (iii) training load (measures related to psychophysiological or physical load); and/ or (iv) injury occurrence.

Quality assessment / Risk of bias analysis: Methodological assessment process was performed by two authors (MRG and FMC) using Downs \& Black assessment criteria for both randomized and non-randomized studies looking studies eligible for inclusion. Each article was assessed based on 27 specific criteria (see Table 3). Each question is scored as 0 (poor quality) or 1 
(good quality), with exception of question 5 ("clear description of principal confounders") that is scored from 0 (not satisfying) to 2 (fully satisfying). Therefore, a maximum of 28 points can be scored for each article. The quality of the article was classified based on the following thresholds: (i) poor (< 14 points); (ii) fair (14-18 points); (iii) good (19-23 points); and (iv) excellent (24-28 points). Any disagreement was discussed and solved by consensus decision. Each item was evaluated using numerical characterization.

Strategy of data synthesis: The following information was extracted from the included original articles: (i) study design; (ii) goals of the study; (iii) characteristics of the participants (e.g., age; number; sex; competitive level); (iv) measures of sleep; (v) measures of athletic performance, match-running performance, training load and athletic injuries.

Subgroup analysis: None.

Sensitivity analysis: None.

Language: English.

Country(ies) involved: Portugal; Spain.

Keywords: football; athletic performance; athletic injuries; load monitoring; sleep.

Contributions of each author:

Author 1 - Filipe Manuel Clemente.

Author 2 - José Afonso.

Author 3 - Júlio Costa.

Author 4 - Rafael Oliveira.

Author 5 - José Pino-Ortega.

Author 6 - Markel Rico-González. 